\title{
Mechanism of Fatty Acid Synthase in Drug Tolerance Related to Epithelial-mesenchymal Transition of Breast Cancer
}

\author{
Jun-Qin Li ${ }^{\circledR}$, Hui Xue ${ }^{\star}$, Lan Zhou, Li-Hua Dong, Da-Peng Wei, Hua Li*
}

\begin{abstract}
Objective: The mechanism of action of fatty acid synthase (FASN) in drug tolerance of breast cancer cells with epithelial-mesenchymal transition (EMT) features was investigated. Methods: The breast cancer cell line MCF-7-MEK5 with stably occurring EMT and tumour necrosis factor- $\alpha$ (TNF- $\alpha$ ) tolerance was used as the experimental model, whereas MCF-7 acted as the control. Tumour cells were implanted into nude mice for in vivo analysis, and cerulenin was used as a FASN inhibitor. RT-PCR, real-time quantitative PCR and Western blot were employed to detect the expression of FASN, TNFR-1, TNFR-2, Wnt-1, $\beta$-catenin and cytC at the RNA and protein levels. Results: Compared with MCF-7, TNFR-1 expression in MCF-7-MEK5 was slightly changed, TNFR-2 was decreased, and FASN, Wnt-1, $\beta$-catenin and cytC were increased. The expression of Wnt-1 and $\beta$-catenin in MCF-7-MEK5 decreased after cerulenin treatment, whereas cytC expression increased. Conclusions: The important function of FASN in the drug tolerance of breast cancer may be due to the following mechanisms: FASN downregulated TNFR-2 expression through lipid rafts to make the cells less sensitive to TNF- $\alpha$, and simultaneously activated the Wnt-1/ß-catenin signalling pathway. Thus, cytC expression increased, which provided cells with anti-apoptotic capacity and induced drug tolerance.
\end{abstract}

Keywords: Breast cancer - EMT - FASN - TNF- $\alpha$ - TNFR - Wnt - $1 / \beta$ - catenin

Asian Pac J Cancer Prev, 15 (18), 7617-7623

\section{Introduction}

Drug tolerance is one of the reasons why cancer is difficult to treat. Many patients acquire therapy tolerance after several drug treatments, and these resistant therapy traits are associated with epithelial-mesenchymal transition (EMT) in various tumours (Mani et al., 2008; Han et al., 2014). Wang et al (2009) found that gemcitabine tolerance for pancreatic cancer cells is closely related to EMT, and the Notch signalling pathway is indispensable in the occurrence of EMT . They also found that knockdown of DUSP4 reverses EMT in MCF-7/ADR cells (adriamycinresistant), and increases the chemosensitivity of MCF-7/ ADR cells to doxorubicin (Lin et al., 2013). Zhou et al. (2008) found that the overexpression of MEK5 induces EMT and tumour necrosis factor- $\alpha$ (TNF- $\alpha)$ tolerance in MCF-7 cells. Thus, studying the mechanism for drug resistance in EMT cells will benefit tumour treatment.

Several studies showed that fatty acid synthase (FASN) has a major function in acquiring resistant phenotype, whereas the FASN level is associated with the presentation of inherent resistant phenotype in hepatocellular carcinoma (Meena et al., 2013). The FASN inhibitor osthole can abrogate HGF-induced cell scattering, migration and invasion in MCF-7 breast cancer cells (Hung et al., 2011). Moreover, reducing FASN expression increases drug sensitivity in breast cancer cell lines MCF7 and MDA-MB-468, but not in normal mammary epithelial cell line MCF10A1 (Liu et al., 2008). The present study showed that the overexpression of MEK-5 and cell apoptosis in breast cancer cell line MCF7-MEK5 with EMT characteristics and TNF- $\alpha$ tolerance occurred when the cells were treated by FASN inhibitor in the overexpression of FASN. Thus, we hypothesised that FASN was closely related to the drug tolerance of breast cancer cell line MCF-7-MEK5. However, the mechanism of action remains unclear.

MCF-7-MEK5 cells have TNF- $\alpha$ tolerance (Zhou et al., 2008). TNF- $\alpha$ is a multifunctional cytokine with biological effects that can cause diseases, such as inflammation, killing or inhibition of tumour cells and proliferation and differentiation of tumour cells, which produces a double-edged sword effect. TNF- $\alpha$ can combine with two receptors, namely, type $1 \mathrm{TNF}$ Receptor (TNFR-1) and type 2 TNF Receptor (TNFR2). TNFR-1 is expressed in most tissues, and both the membrane-bound and soluble trimeric TNF receptors can activate these receptors. The loss of either TNF or TNFR-2 can prevent the accumulation of Treg cells and result in a less tolerogenic environment, which enables the immune system to control B16F10 tumour metastasis and growth (Chopra et al., 2013). Furthermore, studies on

Department of Anatomy, School of Basic and Forensic Medicine, Sichuan University, Chengdu, China ${ }^{\star}$ Equal contributors $*$ For correspondence: lihua_scu@scu.edu.cn 
lipid catabolism of mice with deletion TNFR (TNFR-1 (TNFR-1 (-/-), TNFR-2 (TNFR-2 (-/-), (TNFR-1 (-/-) R-2 $(-/-))$ showed that the TNF- $\alpha$-induced effects on lipolysis mainly acts through TNFR-1 (Sethi et al., 2000). The concentration of soluble receptors for TNF may serve as an additional predictor of the presence and clinical course of disease in patients with prostate cancer (Sethi et al., 2000). TNFR is a membrane-bound receptor tyrosine kinase (RTK) associated with fatty acid metabolism, which indicates that this receptor may be possibly linked to the function of FASN. However, no related studies have been reported at present.

We hypothesised that FASN determined the function of anchoring to RTKs in the cell membrane, including TNFR, by affecting the cell membrane structure of lipid rafts. If a relationship exists between FASN and TNFR, the mechanism by which cerulenin suppresses MCF-7MEK5 apoptosis, which results from FASN, should be investigated. In addition, the key signalling molecules involved in this process should be determined.

In the EMT process of MCF-7-MEK5 in breast cancer cells, E-cadherin expression significantly decreases (Zhou et al., 2008). E-cadherin is a class of $\mathrm{Ca}^{2+}$-dependent transmembrane adhesion receptors with homogeneous affinity, and adheres among epidermal cells as a marker molecule. The intracellular region of E-cadherin can connect with $\beta$-catenin, which interacts with the cytoskeleton through $\alpha$-catenin to stabilise the connections among epithelial cells, and has an important function in the maintenance of epithelial cell morphology and cell adhesion, in which $\beta$-catenin is a major regulatory molecule (Douglas et al., 2010). Studies showed that growth factors could regulate the stability of E-cadherin/ $\beta$-catenin complexes by phosphorylation and dephosphorylation of $\beta$-catenin. The intracellular domain of epidermal growth factor receptor (EGFR) is closely bound to $\beta$-catenin. Given that the tyrosine residues of EGFR are phosphorylated, $\beta$-catenin is dephosphorylated when bound to the ligand EGF. The stability of the E-caherin/ $\beta$-catenin compound decreases, which negatively affects the E-cadherin-mediated adhesion function. Thus, adhesion reduction and migration increase among tumour cells, which consequently increase the ability of tumour invasion and metastasis (Tian et al., 2011). The Wnt- $1 / \beta$-catenin signalling pathway is also related to anti-cancer treatment. The Wnt-1 signalling pathway can inhibit the induced apoptosis of cancer therapy from drugs by blocking cytochrome $\mathrm{C}$ (cytC) release, then inhibiting the mitochondrial apoptosis pathway, such as caspase-9 activation (Chen et al., 2001), which is associated with various occurrences for tumour EMT (Han et al., 2013; Kim et al., 2013). CytC, which is derived from the mitochondrial apoptosis signalling molecules (Huttemann et al., 2012), is generally considered as a molecular marker of apoptosis (Xu et al., 2013). However, some studies showed that cytC is also associated with cell drug tolerance. Shaoqiong Chen et al found that the Wnt-1 signalling pathway can induce drug tolerance of mouse fibroblast cells by inhibiting the release of cytC (Chen et al., 2001). Thus, we hypothesised that Wnt- $1 / \beta$-catenin had an important function in EMT and drug tolerance of MCF-7-MEK5 by regulating the cytC signalling system, and was closely associated with the overexpression of FASN.

The breast cancer cell line MCF-7-MEK5 exhibits TNF- $\alpha$ tolerance and EMT characteristics. Early preliminary experiments also showed that FASN expression in MCF-7-MEK5 cells and tumour tissues significantly increases than that in MCF-7. In the present study, changes in the expression of TNFR, Wnt-1, $\beta$-catenin and cytC were observed after FASN inhibition to determine the mechanism of FASN in drug tolerance of MCF-7-MEK5. The relationship between these changes and FASN was also investigated.

\section{Materials and Methods}

\section{Cell culture}

MCF-7 and MCF-7-MEK5 cells were provided by the Huaxi Preclinical and Forensic Medicine Department Immunology Section of Sichuan University. The adherent cells were cultured in an incubator with $10 \%$ foetal bovine serum containing RPMI-1640 medium at $37^{\circ} \mathrm{C}, 5 \% \mathrm{CO}_{2}$ and saturated humidity.

\section{Animals and grouping}

A total of 18 five-week-old, female, specific pathogenfree BALB/c nude mice (weight ranging from $18 \mathrm{~g}$ to $20 \mathrm{~g}$ ) were provided by the Sichuan University Huaxi Medical Experimental Animal Centre. The mice were randomly divided into two groups, with nine mice in each group. These two main groups were then randomly divided into the experimental group (six mice) and control group (three mice). This study was carried out in strict accordance with the recommendations in the Guide for the Care and Use of Laboratory Animals of the National Institutes of Health. The animal use protocol has been reviewed and approved by the Institutional Animal Care and Use Committee (IACUC) of Sichuan University.

\section{Tumour model preparation}

MCF-7 and MCF-7-MEK5 cells were digested, rinsed with Hanks solution and adjusted to a cell concentration of $1 \times 10^{7} / \mathrm{mL}$. Each nude mouse was vaccinated with 0.1 $\mathrm{mL}$ of the solution under the breast pads in the chest walls (MCF-7 cells was injected for the A group, and MCF-7MEK5 cells for the B group). The food for the mice was placed in a layer and fluid hood. When the tumour volume reached $100 \mathrm{~mm}^{3}$, six mice were randomly selected as the experimental group and injected intraperitoneally with $160 \mathrm{mg} / \mathrm{d} / \mathrm{kg}$ cerulenin (BML-G237-0025, Biomol, NY, USA) for $10 \mathrm{~d}$. The mice were killed after $5 \mathrm{~d}$ and their organs were removed.

\section{Real-time quantitative PCR}

Tripure (1667165001, Roche, Basel, Switzerland) was added to cells or tissues to extract the total RNA, and then reversely transcribed into cDNA (\#k1621, Fermentas, Burlington, Canada). The primers were designed using online software Primer 3, and the primer sequences are shown in Table 1 . The specificity of the primer sequence was confirmed using Basic Local Alignment Search 
Table 1. Primers Involved in RT-PCR

\begin{tabular}{|c|c|c|c|}
\hline \multicolumn{2}{|l|}{ Gene name } & Primer sequence $\left(5^{\prime} \rightarrow 3^{\prime}\right)$ & Product length (bp) \\
\hline FASN & $\mathrm{F}$ & CTTCCGAGATTCCATCCTACGC & 131 \\
\hline \multirow[t]{2}{*}{ TNFR-1 } & $\mathrm{F}$ & GGCATCCAGCAGGATATCAA & 159 \\
\hline & $\mathrm{R}$ & CATAAGGACCAGGGACAGCA & \\
\hline \multirow[t]{2}{*}{ TNFR-2 } & $\mathrm{F}$ & TGGACTGATTGTGGGTGTGA & 136 \\
\hline & $\mathrm{R}$ & CTTATCGGCAGGCAAGTGA & \\
\hline \multirow[t]{2}{*}{ Wnt-1 } & $\mathrm{F}$ & CCTCCACGAACCTGCTTACA & 108 \\
\hline & $\mathrm{R}$ & TCCCCGGATTTTGGCGTATC & \\
\hline \multirow[t]{2}{*}{ B-Catenin } & $\mathrm{F}$ & AAGCTTCCAGACACGCTATCA & 200 \\
\hline & $\mathrm{R}$ & CACTGGTGAACCAAGCATTTT & \\
\hline \multirow[t]{2}{*}{ CytC } & $\mathrm{F}$ & AAAGATGGGTTACCTGCGACT & 162 \\
\hline & $\mathrm{R}$ & ACACGGCCTTTTCGTAAATCT & \\
\hline \multirow[t]{2}{*}{ GAPDH } & $\mathrm{F}$ & CTGCCCCCTCTGCTGATG & 150 \\
\hline & $\mathrm{R}$ & TCCACGATACCAAAGTTGTCAT & \\
\hline
\end{tabular}

Tool (NCBI, USA), which was synthesised by Shanghai Biological Engineering Company. PCR was carried out in accordance with the instructions of the PCR kit (\#k0221, Fermentas). In brief, denaturation was carried out at $95^{\circ} \mathrm{C}$ for 1 minute, followed by 40 cycles $\left(95^{\circ} \mathrm{C}\right.$ for $15 \mathrm{~s}$, $60^{\circ} \mathrm{C}$ for $30 \mathrm{~s}$ and $72^{\circ} \mathrm{C}$ for $30 \mathrm{~s}$ ), with a primer annealing temperature of $60^{\circ} \mathrm{C}$. The experimental data were analysed using the ${ }^{\Delta \Delta} \mathrm{Ct}$ method. All independent experiments were repeated thrice. GraphPad Prism 5 software was used, and data analysis was performed through t-test. Data were plotted using mean \pm SD.

\section{Western blot}

Lysis buffer (KGP2100, KeyGEN BioTECH, Nanjing, China) was added to cells or tissues. The mixture was then vortexed, incubated at $4^{\circ} \mathrm{C}$ for 30 minutes and centrifuged at $13,000 \times \mathrm{g}$ at $4^{\circ} \mathrm{C}$ for 15 minutes. The supernatant was obtained to determine the protein quantity by the bicinchoninic acid method (KGSK3051, KeyGEN BioTECH, Nanjing, China). The samples were boiled for 5 minutes to denature the proteins, and the samples were loaded on sodium dodecyl sulphate-polyacrylamide gel electrophoresis (SDS-PAGE). The damp-dry SDSPAGE was transferred to the nitrocellulose membranes, and the film was sealed with $5 \mathrm{~g} / \mathrm{L}$ skimmed milk. The first anti-FASN (273 kDa, ab22759, Abcam; 1:5000), rabbit anti-human TNFR-1 polyclonal antibody (36 kDa, bs-4962R, Bioss; 1:500), rabbit anti-human TNFR-2 polyclonal antibody (52 kDa, bs-7678R, Bioss; 1:500), rabbit anti-human wnt-1 polyclonal antibody (42 kDa, bs-1947R, Bioss; 1:500), rabbit anti-human $\beta$-catenin polyclonal antibody (86 kDa, bs-1165R, Bioss; 1:500) and rabbit anti-human cytC polyclonal antibody (12 kDa, bs8166R, Bioss; 1:500) were used for overnight incubation at $4^{\circ} \mathrm{C}$. The samples were fully rinsed with PBS-Tween $(0.05 \%)$, added with horseradish peroxidase-conjugated secondary antibody (ZB2301, anti-rabbit; ZB2305, antimouse; ZSGB-BIO) and incubated at $37^{\circ} \mathrm{C}$ for 2 hours. The membrane was then washed, cleaned and coloured by enhanced chemiluminescence (KGP112, KeyGEN BioTECH).

\section{Statistical analysis}

Real-time PCR was carried out in triplicate. The average of three experiments was used for statistical analysis and data are presented as mean \pm SD. Data were analysed by GraphPad Prism version 5.0 for Windows (GraphPad Software, San Diego, CA, USA). Student's $\mathrm{t}$-test was used to detect the difference between groups, and $p<0.05$ was considered statistically significant.

\section{Results}

Expression of FASN

The RT-PCR results show that FASN expression in breast cancer cell line MCF-MEK5 and tumourigenic tissues increased than that in MCF-7 (Figure 1A). The real-time quantitative $\mathrm{PCR}$ results show that the increased multiples of FASN expression in MCF-7-MEK5 were $39.12 \pm 8.21$ (in vitro) and 43.78 \pm 10.23 (in vivo) (Figure $1 B$ ), with a significant difference compared with that in the control group. Western blot results further confirmed the findings at the protein level, in which FASN expression in both MCF-MEK5 cells and tumour tissues was upregulated (Figure 1C). These results indicate that the overexpression of FASN had an important function in breast cancer cell line MCF-7-MEK5 with TNF- $\alpha$ tolerance.

Expression changes in TNFR-1,TNFR-2, Wnt-1, $\beta$-catenin and $c y t C$

The expression levels of TNFR-1, TNFR-2, Wnt-1,
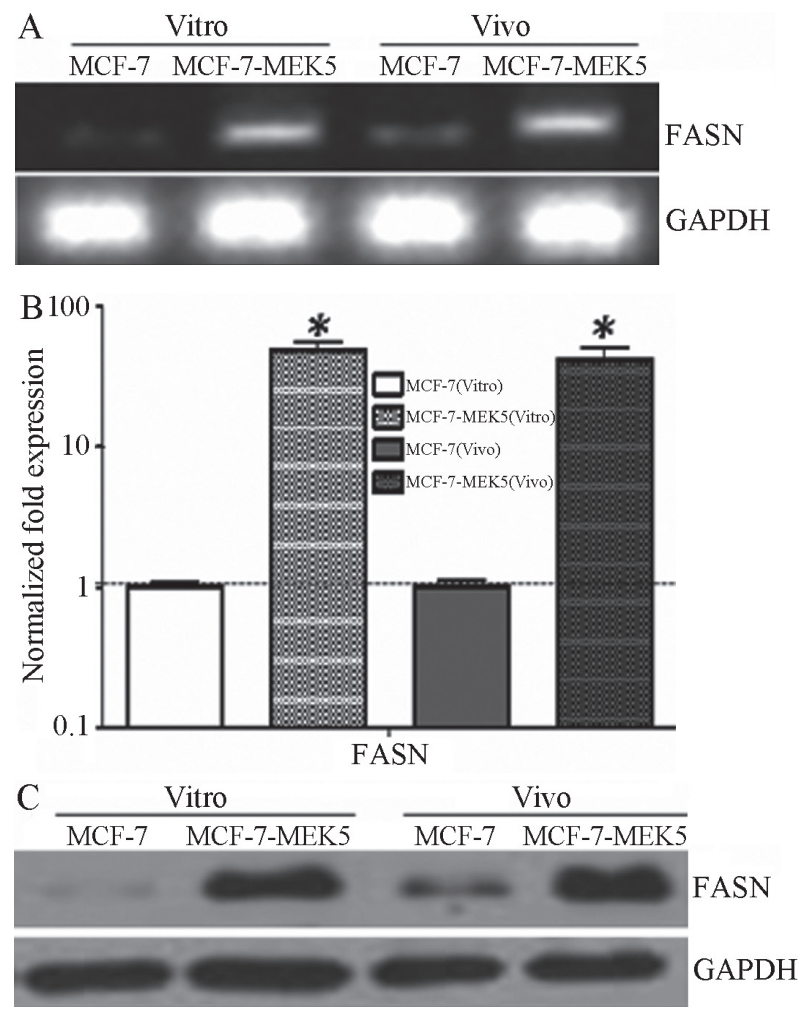

Figure 1. Expression Levels of FASN in MCF-MEK5 and The Situ Tumor Tissues Significantly Increased. A. RT-PCR results showed that FASN mRNA expression in MCF7-MEK5 cells and in situ tumor tissues upregulated compared with that in MCF-7 cells. B. Real-time PCR results showed that FASN mRNA expression in MCF-7-MEK5 cells and in situ tumor tissues grew exponentially compared with that in MCF-7 cells, and the differences had statistically significant $(* P<0.05)$. C. Western Blot test results further confirmed FASN mRNA expression in MCF-7-MEK5 cells and in situ tumor tissues upregulated at the protein level. Note: GAPDH was an internal control, $*=P<0.05$ indicated significant difference 

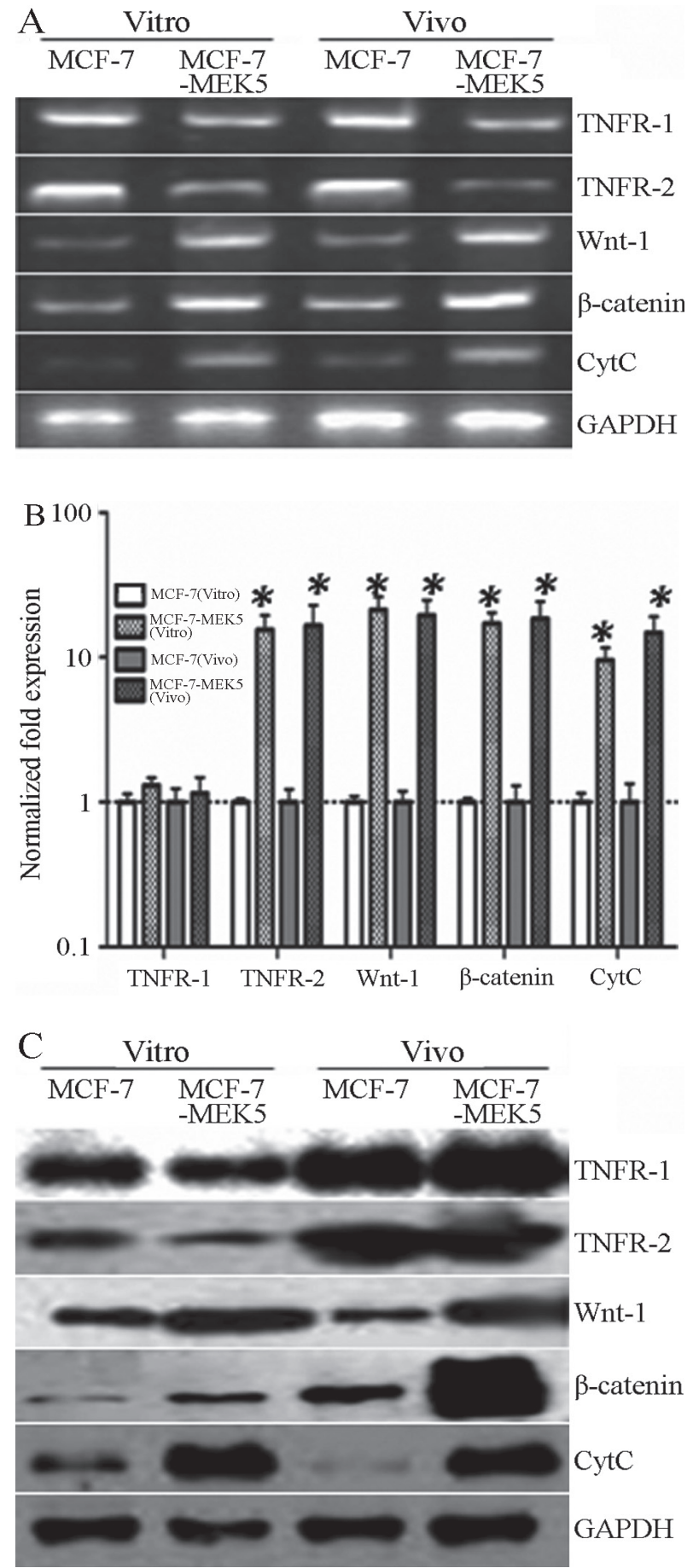

Figure 2. Expression Changes of TNFR-1, TNFR-2, Wnt-1, $\beta$-catenin and CytC in MCF-7 and MCF-7MEK5 in vivo. A. RT-PCR results showed that TNFR-1 mRNA expression in cells and tissues of the two groups, TNFR2 expression in MCF-7-MEK5 cells and in situ tumor tissues downregulated, and Wnt-1, B-Catenin, CytC upregulated. B. Real-time PCR results showed that TNFR-1 mRNA expression in the two groups, the expression of TNFR-2 mRNA in MCF-7-MEK5 cells and in situ tumor tissues downregulated exponentially compared with that in MCF-7 cells, while the expression of Wnt-1, $\beta$-catenin and CytC upregulated exponentially with statistically significance $(* P<0.05)$. C. Western Blot test results further confirmed TNFR-1 expression in the two cells and tissues had no significant differences at the protein level, the expression of TNFR-2 decreased, and Wnt$1, \beta$-catenin, CytC increased. Note: GAPDH was an internal control, ${ }^{*}=P<0.05$ indicated significant difference $\beta$-catenin and cytC in breast cancer cell lines MCF-7 and MCF-7-MEK5, as well as their tumourigenic tissues, were detected. The results show that TNFR-1 expression in the two nucleus tissues had no difference. However, compared with MCF-7, TNFR-2 expression in MCF-7-MEK5 cells and tumour tissues decreased, whereas the expression of Wnt-1, $\beta$-catenin and cytC in cells and tumour tissues of MCF-7-MEK5 increased (Figure 2A). The real-time PCR results show that the expression multiples of TNFR-1 in MCF-7MEK-5 cells and tumour tissues were $1.15 \pm 0.03$ (in vitro) and $1.39 \pm 0.17$ (in vivo) higher than those of MCF-7, respectively. TNFR-2 expression in MCF-7MEK5 decreased than that in MCF-7 by $15.56 \pm 3.96$ (in vitro) and 16.576 .36 (in vivo) times, respectively; Wnt-1 expression increased by $21.33 \pm 4.94$ (in vitro) and $19.54 \pm 5.34$ (in vivo) times; $\beta$-catenin expression increased by $17.18 \pm 3.23$ (in vitro) and $8.46 \pm 5.73$ (in vivo) times; and cytC expression increased by $55 \pm 2.12$ (in vitro) and $14.77 \pm 4.32$ (in vivo) times (Figure 2B). Western blot confirmed the expression of the aforementioned factors at the protein level (Figure 2C). These results suggest that drug tolerance of breast cancer cell line MCF-7-MEK5 to TNF- $\alpha$ could be associated with the decreased expression of TNFR-2, activation of Wnt- 1 and $\beta$-catenin signaling molecules and overexpression of FASN.
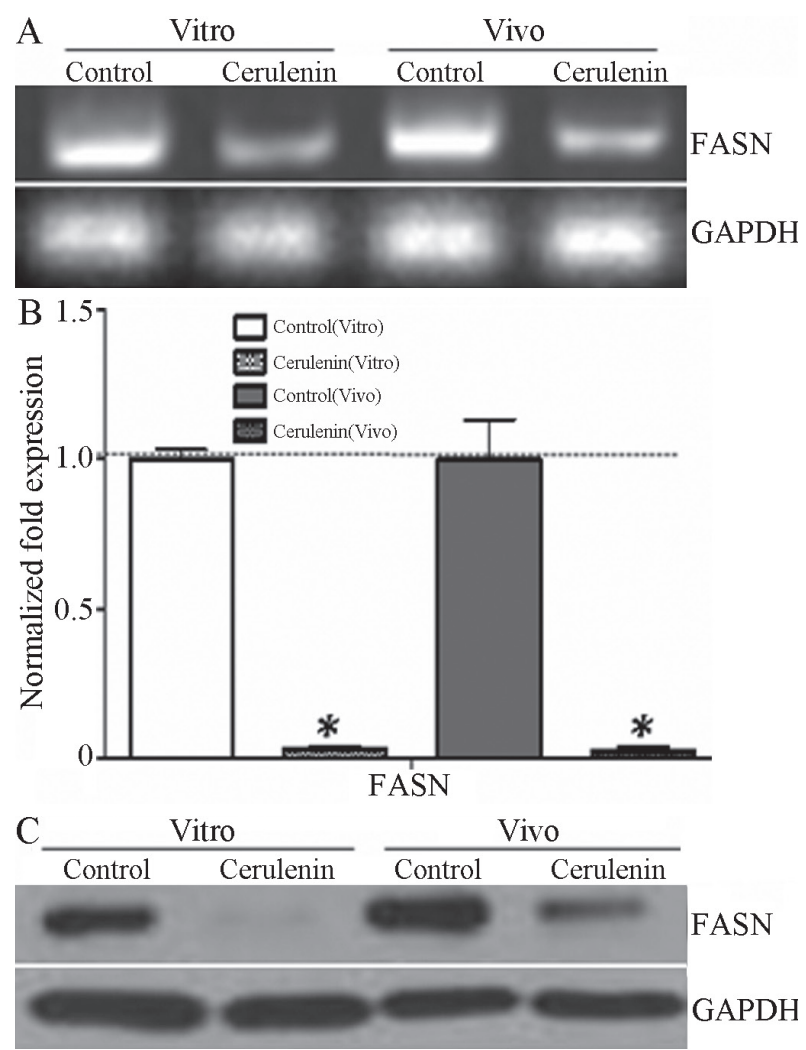

Figure 3. Inhibition of Cerlenin to FASN Expression in MCF-7-MEK5. A. RT-PCR results showed that the expression of FASN mRNA significantly reduced in cerulenin treated groups. B. Real time PCR results showed that the expression amount of FAS mRNA in the drug treatment groups decreased exponentially, the expression levels before and after treatment were significantly different $(* P<0.05)$. C. Western Blot results further confirmed that the expression of FASN significantly downregulated in cerulenin treated MCF-7-MEK5 cells and in situ tumor tissues. Note: GAPDH was an internal control, $*=P$ $<0.05$ indicated significant difference 

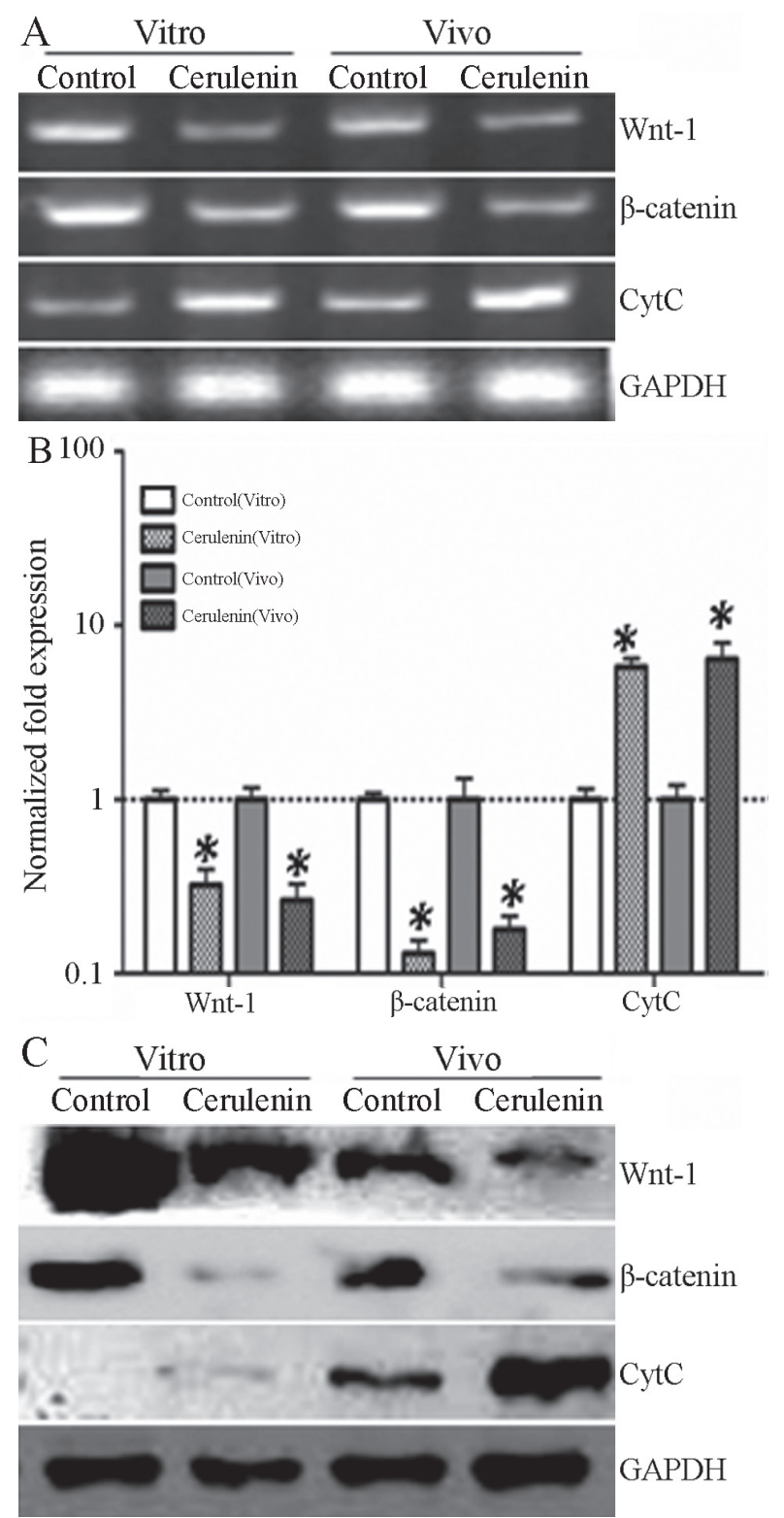

Figure 4. Expression Changes of Wnt-1, $\beta$-catenin and CytC in MCF-7-MEK5 after Cerulenin Treatment. A. RT-PCR results showed that the expression of FASN mRNA significantly reduced in cerulenin treated groups. B. Real time PCR results showed that the expression amount of FAS mRNA in the drug treatment groups decreased exponentially, the expression levels before and after treatment were significantly different $(* P<0.05)$. C. Western Blot results further confirmed that the expression of FASN significantly downregulated in cerulenin treated MCF-7-MEK5 cells and in situ tumor tissues. Note: GAPDH was an internal control, $*=P<0.05$ indicated significant difference

\section{Significant inhibition of cerulenin to FASN expression}

After treating breast cancer cell line MCF-7-MEK5 with TNF- $\alpha$ tolerance and in situ tumour tissues with cerulenin, FASN mRNA expression decreased (Figure 3A). FASN mRNA expression decreased exponentially in the treatment groups than that in the control group $[0.0532 \pm 0.0163$ (in vitro) and $0.0253 \pm 0.0094$ (in vivo), with a significant difference between the two groups Figure 3B). Western blot results further confirmed the downregulated expression of FASN in the drug treatment group at the protein level (Figure 3C).
Expression changes in Wnt-1, $\beta$-catenin and cytC in $M C F$ 7-MEK5 after cerulenin treatment

After treating breast cancer cell line MCF-7-MEK5 with TNF- $\alpha$ tolerance and in situ tumour tissues with cerulenin, the expression levels were detected. The results show that the expression of Wnt- 1 and $\beta$-catenin was downregulated after cerulenin treatment of nucleus organisation, and cytC expression increased (Figure 4A). Wnt-1 mRNA expression decreased by $0.322 \pm 0.075$ (in vitro) and $0.263 \pm 0.064$ (in vivo) times than that before drug treatment. $\beta$-Catenin mRNA expression decreased by $0.1300 \pm 0.0251$ (in vitro) and $0.179 \pm 0.035$ (in vivo) times, whereas cytC mRNA expression increased by $5.77 \pm 0.68$ (in vitro) and $6.39 \pm 1.58$ (in vivo) times. The expression of these factors exhibited significant differences $(*, p<0.05)$ before and after cerulenin treatment (Figure 4B). Western blot further confirmed that the expression of Wnt-1 and $\beta$-catenin decreased in the drug treatment group at the protein level, whereas cytC expression increased in the drug treatment group (Figure 4C). These results indicate that cerulenin not only had an inhibitory effect on FASN, but also inhibited the expression of Wnt-1 and $\beta$-catenin, activated cytC and promoted MCF-7-MEK5 cell apoptosis. In the drug tolerance regulation of FASN on breast cancer cell line MCF-7-MEK5, Wnt-1, $\beta$-catenin and cytC worked as the downstream signalling molecules.

\section{Discussion}

The mechanism of tumour drug tolerance is highly complex, and the improvement of the anti-apoptotic ability of cells may be one of the important mechanisms. Apoptosis is a normal process of programmed cell death, and has an important function in normal human embryogenesis and organogenesis. However, the deficiency of apoptotic cells caused by external environmental factors or genetic factors is one of the factors for tumourigenesis. The cell cycle cannot be arrested in G1 phase if the p53 protein in tumour cells have mutated or are missing, thereby leading to drug tolerance because of a lack of apoptotic cells (Foster et al., 2012). Heinlein et al (2008) constructed p53 mutant mice, and found that p53 mutations induce breast cancer cell proliferation without affecting the stability of the genome. p53 can also induce tumour cell apoptosis by downregulating MSP/Mst1. Lung cancer H1299 cells can produce stronger drug tolerance using specific siRNA to interfere with endogenous MSP (Mihara et al., 2003). The present study mainly focused on the anti-apoptotic mechanisms to discuss the drug tolerance mechanism of breast cancer.

Zhou et al (2008) confirmed that breast cancer cell line MCF-7-MEK5 with transfected MEK5 has a stable EMT phenotype, the induced effect of MEK5 is unaffected by carriers and cells with TNF- $\alpha$ tolerance are associated with EMT. Our previous studies found that the expression of FASN, which is a key enzyme of synthetic long chain fatty acids, is significantly upregulated in MCF-7-MEK5. Thus, we hypothesised that FASN regulated the function of TNFR anchored in the cell membrane in the drug tolerance of MCF-7-MEK5 by affecting the structure of lipid rafts to produce TNF- $\alpha$ tolerance and anti- 
apoptotic characteristics. After suppressing FASN, the cell sensitivity to TNF- $\alpha$ increased, and apoptosis occurred. In this study, cell viability decreased and apoptosis occurred when the FASN inhibitor cerulenin was used to treat the drug tolerance of MCF-7-MEK5 cells. This result demonstrates that FASN had an important function in the drug tolerance of MCF-7-MEK5 cells, which was similar to our assumption.

Many in vivo and in vitro experiments confirmed that FASN inhibitors, such as trastuzumab (Herceptin) (Urruticoechea et al., 2009) and anti-Her2 therapy (Puig et al., 2011), EEO (ethyl acetate extract of onion) (Wang et al., 2012) can increase sensitivity of breast cancer cells to the drug or other therapies. Through sucrose gradient centrifugation and immunofluorescence methods, Espinoza et al. demonstrated that FASN inhibitor inhibits sphingomyelin synthesis in lipid rafts and increases the sensitivity of cells to Taxol, and the cell sensitisation ability caused by inhibiting FASN can be observed by destroying the lipid rafts (Espinoza et al., 2010). Thus, the expression changes in TNFR-1 and TNFR-2 in our study were observed by inhibiting FASN to explore the mechanisms of action for FASN in the drug tolerance of MCF-7-MEK5 cells.

The results show that the expression difference of TNFR-1 in MCF-7 and MCF-7-MEK5 cells was insignificant, whereas TNFR-2 expression in MCF7-MEK5 cells decreased than that in MCF-7 cells. Studies showed that the TNFR pathway is an important mechanism of apoptosis. Apoptosis is induced by activating the downstream caspase cascade (Xiao et al., 2011), which decreases TNFR-2 expression, and cells lose their apoptosis ability to produce drug resistance. Thus, TNF- $\alpha$ tolerance of MCF-7-MEK5 could be derived from the decrease in TNFR-2 expression, and cells could lose sensitivity to TNF- $\alpha$.

TNFR-2 is located in the cell membrane, and FASN has a regulatory effect on the cell membrane and its proteins. Ramírez Alvarado et al (2012) found that TNF- $\alpha$ can cause the insensitivity of fat cells to insulin, and regulate cholesterol and lipoprotein metabolism. TNF- $\alpha$ needs to bind to its receptor for its functions to be evident. Thus, FASN induced drug tolerance of breast cancer possibly by downregulating TNFR-2 expression, which was consistent with our hypothesis.

The results in this study also show that the expression of Wnt- 1 and $\beta$-catenin increased in cell line MCF-7MEK5 with overexpressed FASN and TNF- $\alpha$ tolerance. The expression of Wnt- 1 and $\beta$-catenin decreased when cerulenin induced the apoptosis of MCF-7-MEK5. Wnt$1 / \beta$-catenin can regulate apoptosis by interacting with TNF receptor Fas (TNF receptor superfamily member 6) (Kim et al., 2013). Michelangelo et al (2008) found that FASN is regulated using the C14-labelled acetate approach, and discovered the palmitoylation state of Wnt-1 and the steady state of downstream $\beta$-catenin in cytoplasm, which indicated that FASN can adjust the expression of Wnt- 1 and $\beta$-catenin. Our experiments further confirmed this view.

Among the apoptosis-related factors, cytC is often considered as a molecular marker of apoptosis, but its expression levels in tumours still show many contradictions. For example, the expression levels of cytC increase in ovarian clear cell adenocarcinoma (Kim et al., 2012) and esophageal squamous cell carcinoma (Gao et al., 2012), which can induce cells to produce poor prognosis of anti-apoptotic ability and tumour drug resistance.However, the expression decreases in cancerous endometrial cells (Poonyachoti and Deachapunya, 2012) and human non-small cell lung cancer cell lines (Xu et al., 2013) than that in the adjacent tumour tissues. The sensitive of pancreatic cancer cells SW1990 to drug TNF apoptosis inducing ligand (TRAIL) 4 may be associated with CytC (Zhao et al., 2011). Our results show that cytC expression in MCF-7-MEK5 cells increased than that in MCF-7. The Wnt-1 signalling pathway can induce mouse fibroblast cells to produce drug tolerance by inhibiting the release of cytC (Chen et al., 2001). Therefore, cytC possibly regulated cell tolerance via the downstream molecules of the Wnt-1 signalling pathway.

In summary, FASN was a key factor in the drug tolerance of breast cancer, and possibly regulated cell drug tolerance through the following mechanisms: FASN downregulated TNFR-2 expression in lipid rafts to cause cell insensitivity to TNF- $\alpha$, and simultaneously activated the Wnt- $1 / \beta$-catenin signalling pathways, thereby upregulating cytC to result in cell anti-apoptotic ability and induce drug tolerance. Moreover, MCF-7-MEK5 cells had TNF- $\alpha$ tolerance, but cerulenin effectively inhibited the proliferation of MCF-7-MEK5 cells, which indicates that patients with TNF- $\alpha$ could be individually treated by inhibiting FASN expression.

\section{References}

Chen S, Denis CG, You ZB, et al (2001). Wnt-1 Signaling inhibits apoptosis by activating b-catenin/T cell factor-mediated transcription. J Cell Biol, 152, 87-96.

Chopra M, Riedel SS, Biehl M, et al (2013). Tumor necrosis factor receptor 2-dependent homeostasis of regulatory $\mathrm{T}$ cells as a player in TNF-induced experimental metastasis. Carcinogenesis, 34, 1296-303.

Espinoza I, Hong PC, Busby R, Ruth L (2010). Inhibition of fatty acid synthase (FASN) sensitizes breast cancer cells to chemotherapy and disrupts the lipid rafts. Cancer Res, 70, p4-03-01.

Fiorentino M, Zadra G, Palescandolo E, et al (2008). Overexpression of fatty acid synthase is associated with palmitoylation of Wnt-11 and cytoplasmic stabilization of b-catenin in prostate cancer. Lab Invest, 88, 1340-8.

Foster SS, De S, Johnson LK, et al (2012). Cell cycle- and DNA repair pathway-specific effects of apoptosis on tumor suppression. Proc Natl Acad Sci USA, 109, 9953-8.

Gao FL, Song WG, Li WS, et al (2012). Correlation between expression of apoptosis modulators Smac and Cyt C and prognosis in esophageal squamous cell carcinoma. World Chinese J Digestology, 29, 226-8.

Han RF, Ji X, Dong XG, et al (2013). An epigenetic mechanism underlying doxorubicin induced EMT in the human BGC823 gastric cancer cell. Asian Pac J Cancer Prev, 15, 4271-4.

Han XY, Wei B, Fang JF, et al (2013). Epithelial-mesenchymal transition associates with maintenance of stemness in spheroid-derived stem-like colon cancer cells. PLoS One, 8, 73341 .

Heinlein C, Krepulat F, Lohler J, et al (2008). Mutant p53 
$(\mathrm{R} 270 \mathrm{H})$ gain of function phenotype in a mouse model for oncogene-induced mammary carcinogenesis. Int J Cancer, 122, 1701-9.

Hung CM, Kuo DH, Chou CH, et al (2011). Osthole suppresses hepatocyte growth factor (HGF)-induced epithelialmesenchymal transition via repression of the c-Met/Akt/ mTOR pathway in human breast cancer cells. J Agric Food Chem, 5, 9683-90.

Hüttemann M, Lee I, LI G, et al (2012). Phosphorylation of mammalian cytochrome $\mathrm{c}$ and cytochrome $\mathrm{c}$ oxidase in the regulation of cell destiny: respiration, apoptosis, and human disease. Adv Exp Med Biol, 748, 237-64.

Kim MK, Maeng YI, Sung WJ, et al (2013). The differential expression of TGF- $\beta 1$, ILK and wnt signaling inducing epithelial to mesenchymal transition in human renal fibrogenesis: an immunohistochemical study. Int J Clin Exp Pathol, 6, 1747-58.

Kim SK, Foote MB, Huang L (2012). The targeted intracellular delivery of cytochrome $\mathrm{C}$ protein to tumors using lipidapolipoprotein nanoparticles. Biomaterials, 33, 3959-66.

Liu H, Liu Y, Zhang JT (2008). A new mechanism of drug resistance in breast cancer cells: fatty acid synthase overexpression-mediated palmitate overproduction. $\mathrm{Mol}$ Cancer Ther, 7, 263-70.

Liu Y, Du F, Chen W, ET AL (2013). Knockdown of dual specificity phosphatase 4 enhances the chemosensitivity of MCF-7 and MCF-7/ADR breast cancer cells to doxorubicin. Exp Cell Res, 319, 3140-9.

Mani SA, Guo W, Liao MJ, et al (2008). The epithelialmesenchymal transition generates cells with properties of stem cells. Cell, 133, 704-15.

Meena AS, Sharma A, Kumari R, et al (2013). Inherent and acquired resistance to paclitaxel in hepatocellular carcinoma: molecular events involved. PLoS One, 8, 61524.

Micalizzi DS, Farabaugh SM, Ford HL (2010). Epithelialmesenchymal transition in cancer: parallels between normal development and tumor progression. J Mammary Gland Biol Neoplasia, 15, 117-34.

Mihara M, Erster S, Zaika A, et al (2003). p53 has a direct apoptogenic role at the mitochondria. Mol Cell, 11, 577-90.

Poonyachoti S, Deachapunya C (2012). Modulatory effects of phytoestrogens on the expression of Fas ligand and the release of cytochrome $\mathrm{C}$ in normal and cancerous endometrial cells. J Med Assoc Thai, 12, 105-12.

Puig T, Aguilar H, Cufi S, et al (2011). A novel inhibitor of fatty acid synthase shows activity against HER $2+$ breast cancer xenografts and is active in anti-HER2 drug-resistant cell lines. Breast Cancer Res, 13, 131.

Ramirez Alvarado M, Sanchez Roitz C (2012). El factor de necrosis tumoral- $\alpha$, la resistencia a la insulina, el metabolismo de lipoproteinas y la obesidad en humanos. Nutr Hosp, 27, 1751-7 (in Spanish).

Sethi JK, Xu H, Uysal KT, et al (2000). Characterisation of receptor-specific TNFalpha functions in adipocytecell lines lacking type 1 and 2 TNF receptors. FEBS Lett, 469, 77-82.

Tian X, Liu Z, Niu B, et al (2011). E-cadherin/ $\beta$-catenin complex and the epithelial barrier. J Biomed Biotechnol, 2011, 567305

Urruticoechea A, Aguilar H, Cufi S, et al (2009). In vivo and in vitro evidence of antitumor activity of UCM-GI028 against trastuzumab-resistant breast cancer. Cancer Res, 69, 5079.

Wang Y, Tian WX, Ma XF. (2013). Inhibitory effects of onion (Allium cepa L.) extract on proliferation of cancer cells and adipocytes via inhibiting fatty acid synthase. Asian Pac $J$ Cancer Prev, 13, 5573-9.

Wang ZW, Li YW, Kong DJ, et al (2009). Acquisition of Epithelial-Mesenchymal Transition phenotype of gemcitabine-resistant pancreatic cancer cells is linked with activation of Notch signaling pathway. Cancer Res, 69 , 2400-7.

Xiao Y, Li H, Zhang J, et al (2011). TNF- $\alpha$ /Fas-RIP-1-induced cell death signaling separates murine hematopoietic stem cells/progenitors into 2 distinct populations. Blood, 118, 6057-67.

Xu TP, Shen H, Liu LX, Shu YQ (2013). Plumbagin from Plumbago zeylanica L induces apoptosis in human non-small cell lung cancer cell lines through NF- $\varkappa \mathrm{B}$ inactivation. Asian Pac J Cancer Prev, 14, 2325-31.

Zhao B, Li L, Cui K, et al (2011). Mechanisms of TRAIL and gemcitabine induction of pancreatic cancer cell apoptosis. Asian Pac J Cancer Prev, 12, 2675-8.

Zhou CH, Nitschke AM, Xiong W, et al (2008). Proteomic analysis of tumor necrosis factor- $\alpha$ resistant human breast cancer cells reveals a MEK5/Erk5-mediated epithelialmesenchymal transition phenotype. Breast Cancer Res, 10, 105 . 